\title{
An Optical Fiber Measurement System for Blade Tip Clearance of Engine
}

\author{
Jia Binghui and He Lei \\ School of Mechanical Engineering, Nanjing Institute of Technology, Nanjing 211167, China \\ Correspondence should be addressed to Jia Binghui; bhjia@njit.edu.cn
}

Received 10 November 2016; Accepted 6 February 2017; Published 28 February 2017

Academic Editor: Roger L. Davis

Copyright ( 2017 Jia Binghui and He Lei. This is an open access article distributed under the Creative Commons Attribution License, which permits unrestricted use, distribution, and reproduction in any medium, provided the original work is properly cited.

\begin{abstract}
The benefits of reducing the tip clearance have been receiving many scholars' attention all the time, which bring turbine efficiency increasing, emissions reduction, payloads increasing, and mission range abilities extension. In order to gain the blade tip clearance dynamically, a prototype optical fiber measurement system was built and tested based on the rotor test rig. The optical fiber tip clearance measurement system consists of the reflective intensity-modulated optical fiber bundle (sensor), main signal processing unit, high-speed data acquisition card, and a computer. The static performance and dynamic performance experiments were conducted to verify the performance of the system we designed. In addition, the results show that the accuracy of the system is $25 \mu \mathrm{m}$ or better; the stability of the measurement system was evaluated in room temperature. The clearance measurement range is about $5 \mathrm{~mm}$, and sensitivity of the sensor is $0.0733 / \mathrm{mm}$. Furthermore, the typical tip clearance dynamic measurement experiment results show that the system has good dynamic response characteristics as well. The system will provide a new tool for engine health monitoring or fast active tip clearance control.
\end{abstract}

\section{Introduction}

Blade tip clearance is defined as the void between the blade tip and the surrounding casing. Minimization of blade tip clearance in the high-pressure turbine (HPT) and highpressure compressor (HPC) has been a subject of intense research since the inception of gas turbine engine. The benefits of reducing the tip clearance include turbine efficiency increase, emissions reduction, and mission range abilities extension [1]. In addition, the aircraft noise and emissions will be reduced as well, which imply additional environmental advantages [2-4]. Unfortunately, engine loads, high-g turns, and maneuver in combination with high-power operation, aerodynamic loads, and wear mechanisms both contributed asymmetric and axisymmetric changes in tip clearance $[1,5]$. However, the method of building the dynamic changes model of tip clearance to achieve the tip clearance real-time changes is very difficult. A measurement technology is desperately needed to realize the accurate real-time tip clearance.

For blade tip clearance measurement, traditional methods currently make use of capacitive measurement methods, eddy current measurement method, radar-type measurement using microwave electromagnetic waves, and laser Doppler velocimetry. The capacitance sensors are most common. Sarma and Barranger [6] proved a capacitance-type blade tip clearance measurement system using a dual amplifier with ramp/DC inputs and integration. Sheard and Killeen $[7,8]$ described a capacitance based tip clearance measurement system which is used for blade-by-blade tip clearance measurement utilizing a frequency-modulated operating mode under laboratory conditions. Long et al. [9] researched the main accuracy influencing factors when using capacitance tip clearance device. Capacitive sensors are simple and inexpensive but they have a poor frequency response and require iron blade [2]. What is more, the measurement accuracy of capacitance sensor is about $50 \mu \mathrm{m}$; this accuracy is difficult to meet the typical clearance range requirements. Eddy current sensors provide noncontact measurements but have disadvantage to sense only magnetic materials. In addition, they are highly dependent on temperature and the blade tip shape $[2,5,10]$. Microwave interferometric techniques [11] allow real-time tip clearance measurement with accuracy of about 


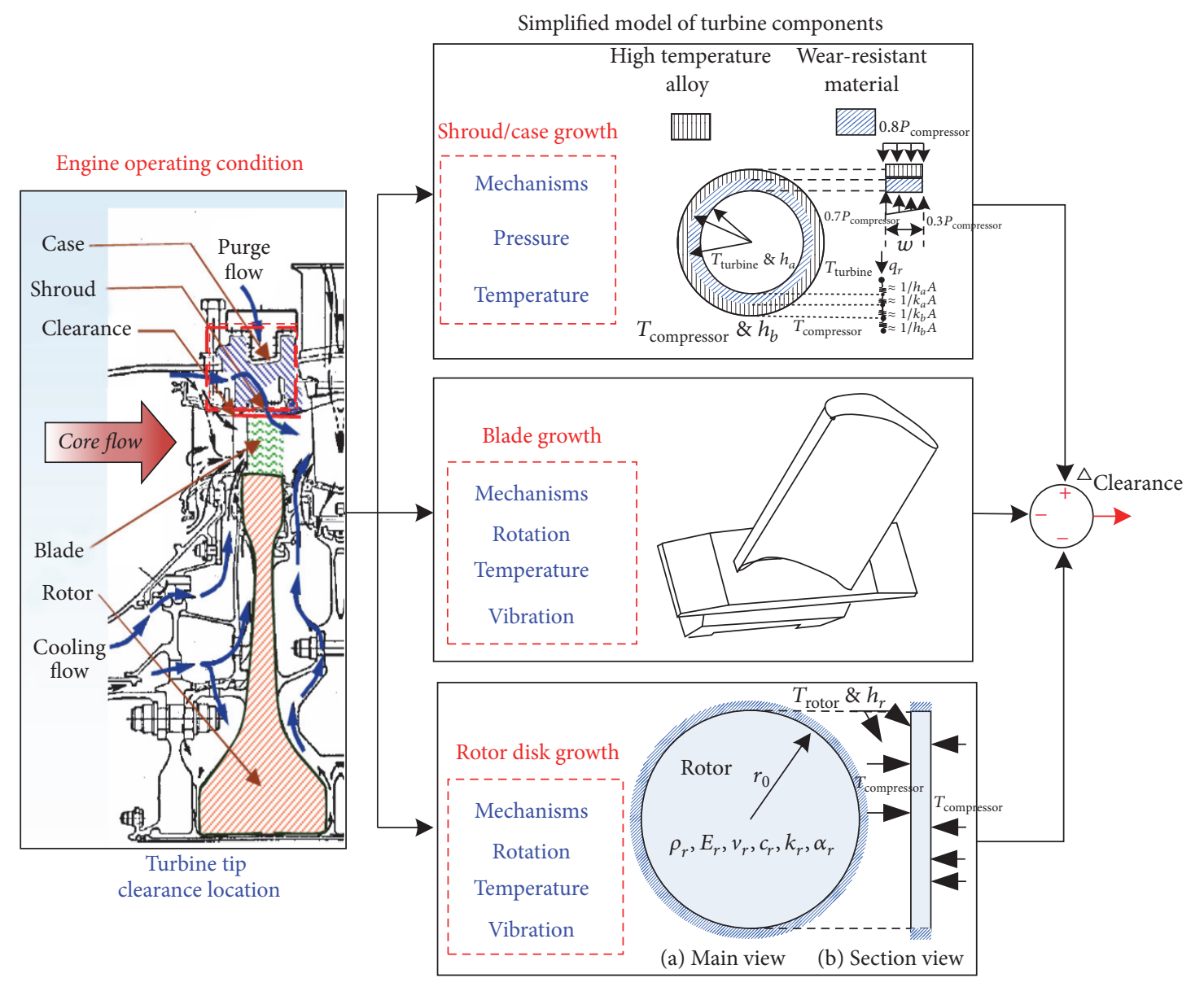

FIGURE 1: Model of tip clearance change.

$20 \mu \mathrm{m}$. Woike et al. [11] report the use of microwave sensor to acquire blade tip clearance being explored at the NASA Glenn Research Center. This type of sensor is beneficial in that it has the ability to operate at extremely high temperature but is very sensitive to the gas flow and the geometry of the sensor environment. On the contrary, optical sensor provides the high available resolution and sensitivity, small size, noncontact measurement and simple instrumentation, and insensitivity to electromagnetic interference [12-15].

In order to achieve the dynamic changes of the gas turbine blade tip clearance based on the mechanisms of HPT tip clearance variation, this paper will describe development and evaluation of a blade tip clearance measurement system using an optical fiber tip clearance sensor. This method is capable of real-time (or near-real-time) tip clearance measurement with accuracy of $25 \mu \mathrm{m}$ or better.

\section{Tip Clearance Measurement Techniques}

2.1. Tip Clearance Change Mechanism. The simplified model of the tip clearance is shown as Figure 1. The left side of the figure shows a cross section of the high-pressure turbine; we can see that the model mainly includes three parts, turbine rotor, blades, and the tip seal (shroud/case). The turbine rotor is mainly made up of the rotor blades, turbine, a turbine shaft, and other parts. The blades are composed of blade body and rabbet that translate the high temperature combustion gas energy into the mechanical work. Turbine casing is an important component of the engine support system; it is usually assumed that the general geometry of the shroud is a ring-like structure, where the inner surface is coated with an abradable material that also functions as a thermal barrier.

Blade tip clearance variations are caused by the displacement or distortion of both the shroud and rotor system over the operating points of the engine. As mentioned previously, the mechanisms behind these variations come from a number of loads on those static and rotating components, such as engine load, flight loads, and aerodynamic and gyroscopic loads. Those loads can produce both axisymmetric and asymmetric tip clearance changes. The uniform radial displacement of shroud/case and the rotating components created by the uniform loads such as centrifugal, thermal, and internal pressure always bring about axisymmetric clearance changes; centrifugal and thermal loads are responsible for the largest radial variations in tip clearance $[1,16-19]$. The nonuniform radial displacement of the stationary structures is usually produced by the nonuniform loads such as gravitational, aerodynamic, and gyroscopic loads that always arouse asymmetric clearance changes. The rotor or blade vibration caused 


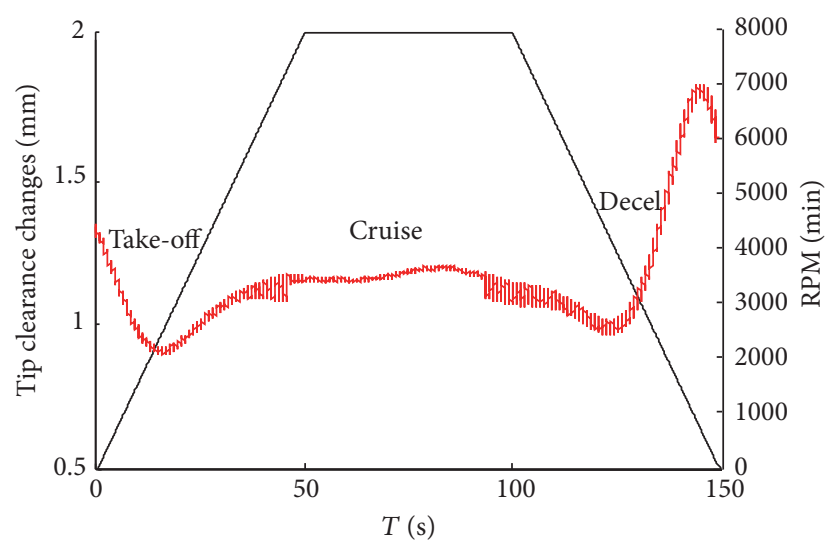

Figure 2: Tip clearance as a function of time over a given mission profile.

by the fight load (thrust, inertial) will produce asymmetric clearance changes.

Considering the above factors, the relative change in the time-varying geometry of each submodel is used to calculate the overall change in tip clearance, as shown in the following equation:

$$
\begin{aligned}
\mathrm{Cl}(t)= & \mathrm{Cl}_{\text {cold }}+r_{\text {shroud }}(t)-r_{\text {rotor }}(t)-l_{\text {blade }}(t) \\
& -v_{\text {vibration }}(t) .
\end{aligned}
$$

Here, $\mathrm{Cl}_{\text {cold }}$ is the initial tip clearance, $\mathrm{Cl}(t), r_{\text {shroud }}(t)$, $r_{\text {rotor }}(t), l_{\text {blade }}(t)$, and $v_{\text {vibration }}(t)$ are, respectively, the total tip clearance, the shroud inner radius, rotor outer radius, blade length, and the rotor vibration displacement as a function of time.

Based on literatures [16-19], the tip clearance and speed as a function of time for a given mission profile due to engine loads and flight loads are shown in Figure 2. The operating points throughout the profile have been labeled take-off, cruise, and decal. We can see that the minimum clearance may occur during the take-off and the deceleration or restarting process shown in Figure 2. In take-off process, with speed increasing, the tip clearance diminished rapidly due to the centrifugal load on the rotor as well as the rapid heating of the turbine blades, which causes the rotating components to grow radially outward, but the shroud/case expands at a much slower rate; then, a minimum gap is produced. Shortly after the case expands, the clearance increases. In deceleration (landing) process, the clearance rapidly increased due to the mechanical unloading of the rotor and blades and the thermal lag of the case as it cools. What is more, the acceleration and deceleration caused by the vibration of the rotor will produce obvious tip clearance changes.

In order to avoid rub, reserved clearance must be set when designing turbine. Unfortunately, the extra space always generates nonoptimal clearance which causes the fuel oil consumption increasing in most of the working conditions. A real-time clearance measurement technology will be conducive to the optimization design of tip clearance and rapid active clearance control implementation.
TABLE 1: Design parameters of the sensor fiber probe.

\begin{tabular}{lc}
\hline Parameters & Value \\
\hline Emitting fiber (EF) & \\
Fiber radius (nucleus) & $150 \mu \mathrm{m}$ \\
Fiber cladding & $15 \mu \mathrm{m}$ \\
NA & 0.22 \\
\hline Receiving fibers (RFs) & \\
Fiber radius (nucleus) & $150 \mu \mathrm{m}$ \\
Fiber cladding & $15 \mu \mathrm{m}$ \\
NA & 0.37
\end{tabular}

Fiber type: standard multimode optical fiber

\begin{tabular}{lc}
\hline Fiber length & $1.5 \mathrm{~m}$ \\
\hline$a$ : diameter of middle fiber bundle & $1050 \mu \mathrm{m}$ \\
\hline
\end{tabular}

2.2. Optical Fiber Displacement Sensor Based on Reflective Light Intensity-Modulated Principle. The measurement system consists of the reflective intensity-modulated optical fiber bundle (sensor), main signal processing unit, high-speed data acquisition card, and a computer, shown in Figure 3. When the measurement system works, the light was sent out from the light source and transmitted to the blade tip surface firstly, and then the receiving fibers received the reflective light and sent it to the photoelectric converter. The size of the received light intensity reflects the distance between the reflector and the fiber probe.

2.2.1. Structure of the Reflective Light Intensity-Modulated Fiber Bundle Probe. The optical fiber structural parameters (fiber core diameter, numerical aperture, and fiber optic axis spacing) have an important influence on the sensor's characteristics. In recent years, fiber optic displacement sensors based on intensity modulation technique have received more considerable research efforts [14], and the sensor model is established based on different assumptions. The doublecircle coaxial optical fiber bundle probe was designed for tip clearance measurement in this paper.

The structure of the reflective intensity-modulated optical fiber bundle composed of a double-circle coaxial optical fiber bundle, as Figure 4 shows. The bundling was divided into three parts: the emitting fiber ( 1 fiber, EF), the inner receiving fibers ( 6 fibers, $\mathrm{RFa}$ ), and the outer receiving fibers (12 fibers, $\mathrm{RFb}$ ). And the design parameters of the sensor fiber bundle are shown in Table 1.

2.2.2. Reflective Light Intensity-Modulated Principle. For the double-circle coaxial optical fiber bundle shown in Figure 4, the distance between $\mathrm{EF}$ of any fiber of RFa is $d$, and the distance between EF of any fiber of RFb is $2 d$. Based on the output optical field distribution function established by Yuan [14] and the intensity modulation mechanism of the two-circle reflective coaxial fiber bundle, The received light 


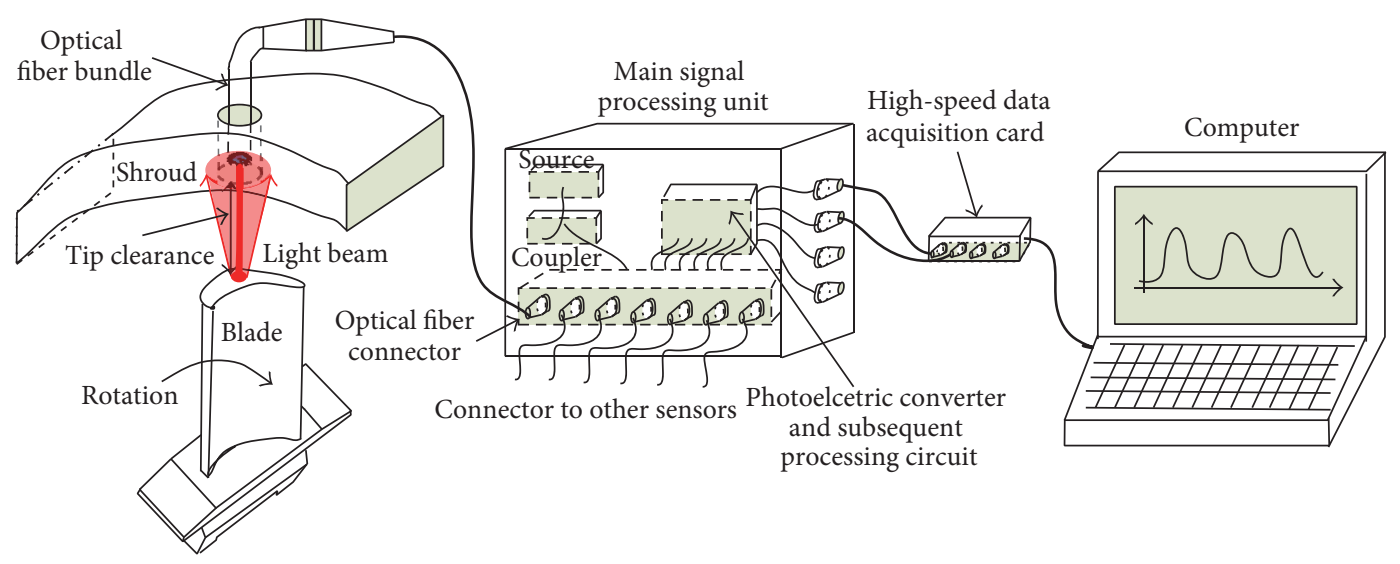

FIGURE 3: Optical fiber tip clearance measurement system.

intensity of $\mathrm{RFa}$ and $\mathrm{RFb}$ can be written, respectively, as follows:

$$
\left.\begin{array}{rl}
I_{1}= & \rho_{1} \cdot \frac{K_{0} K_{1} S_{1} I_{0}}{\pi \sigma^{2} a_{0}^{2}\left[1+\zeta\left(2 z / a_{0}\right)^{3 / 2} \tan \theta_{0}\right]^{2}} \\
& \cdot \exp \left(-\sum_{i} \eta_{i} r_{i}\right) \\
I_{2}= & \rho_{2} \cdot \frac{-d^{2}}{\pi \sigma^{2} a_{0}^{2}\left[1+\zeta\left(2 z / a_{0}\right)^{3 / 2} \tan \theta_{0}\right]^{2}} \\
& \cdot \exp \left(-\sum_{j}^{2} a_{0}^{2}\left[1+\zeta\left(2 z / a_{0}\right)^{3 / 2} \tan \theta_{0}\right]^{2}\right.
\end{array}\right] .
$$

Here, $I_{1}$ is The received light intensity of RFa/cd; $I_{2}$ is the received light intensity of $\mathrm{RFb} / \mathrm{cd}$; $d$ is the distance between $\mathrm{EF}$ and $\mathrm{RFa} / \mu \mathrm{m} ; I_{0}$ is the light intensity by which light source coupled to the EF/cd; $K_{0}$ is the loss of light in optical fiber; $\sigma$ is the parameter related to the optical fiber refractive index profiles, and, for step index optical fiber, $\sigma=1 ; a_{0}$ is the fiber radius $/ \mu \mathrm{m}$; $\zeta$ is the parameter that related to the type of the light source and how the light source coupled to the optical fiber; $\theta_{0}$ is the maximum exit angle of the fiber/rad; $\rho$ is the specular reflectance; $\exp \left(-\sum_{i} \eta_{i} \mathrm{r}_{i}\right)$ is fiber bending losses; $S$ is the fiber capture area $/ \mathrm{mm}^{2}$.

Based on the structure of the double-circle coaxial optical fiber bundle as shown in Figure 4, dividing both receiving light intensity of $\mathrm{RFa}$ and receiving light intensity of $\mathrm{RFb}$, we obtain

$$
M(z)=\frac{I_{2}}{I_{1}} .
$$

Here, $M(z)$ is the ratio of the light intensity received by $\mathrm{RFb}$ and $\mathrm{RFa}$.

Substituting (2) into (3), we gain the following equation:

$M(z)$

$$
\begin{aligned}
= & \frac{\rho_{2} K_{2} S_{2}}{\rho_{1} K_{1} S_{1}} \cdot \frac{\exp \left(-\sum_{j} \eta_{j} r_{j}\right)}{\exp \left(-\sum_{i} \eta_{i} r_{i}\right)} \\
& \cdot \frac{\exp \left[-(2 d)^{2} / a_{0}{ }^{2}\left[1+\zeta\left(2 z / a_{0}\right)^{3 / 2} \tan \theta_{0}\right]^{2}\right]}{\exp \left[-d^{2} / a_{0}{ }^{2}\left[1+\zeta\left(2 z / a_{0}\right)^{3 / 2} \tan \theta_{0}\right]^{2}\right]} .
\end{aligned}
$$

For blade tip plane, the difference of the surface reflectivity can be neglected, and the receiving fiber bundles RFa and $\mathrm{RFb}$ are with the same materials and core area; in addition, the intrinsic loss and the loss caused by fiber bending are nearly equal, so (4) can be simplified as follows:

$$
\begin{aligned}
\rho_{1} & =\rho_{2} \\
S_{1} & =S_{2} \\
K_{1} & =K_{2} \\
\exp \left(-\sum_{i} \eta_{i} r_{i}\right) & =\exp \left(-\sum_{j} \eta_{j} r_{j}\right) .
\end{aligned}
$$

And we obtain the output characteristic function of the fiber bundle:

$$
M(z)=\exp \left\{\frac{-3 \cdot d^{2}}{a_{0}^{2}\left[1+\zeta\left(2 z / a_{0}\right)^{3 / 2} \tan \theta_{0}\right]^{2}}\right\} .
$$

Therefore, the quotient of the irradiance only depends on a constant related to the losses in the optical fibers and it is a function of the distance to the illuminated blade. 


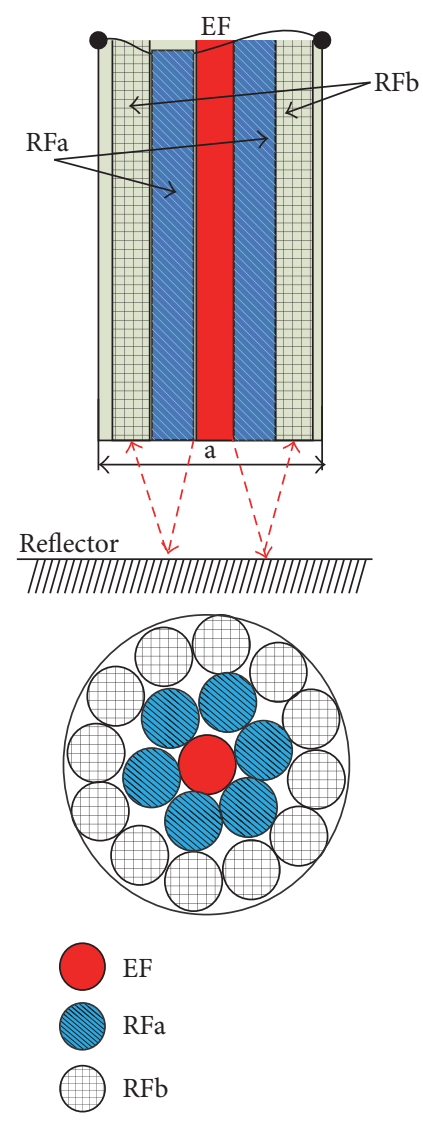

(a) Arrangement of the fiber bundle

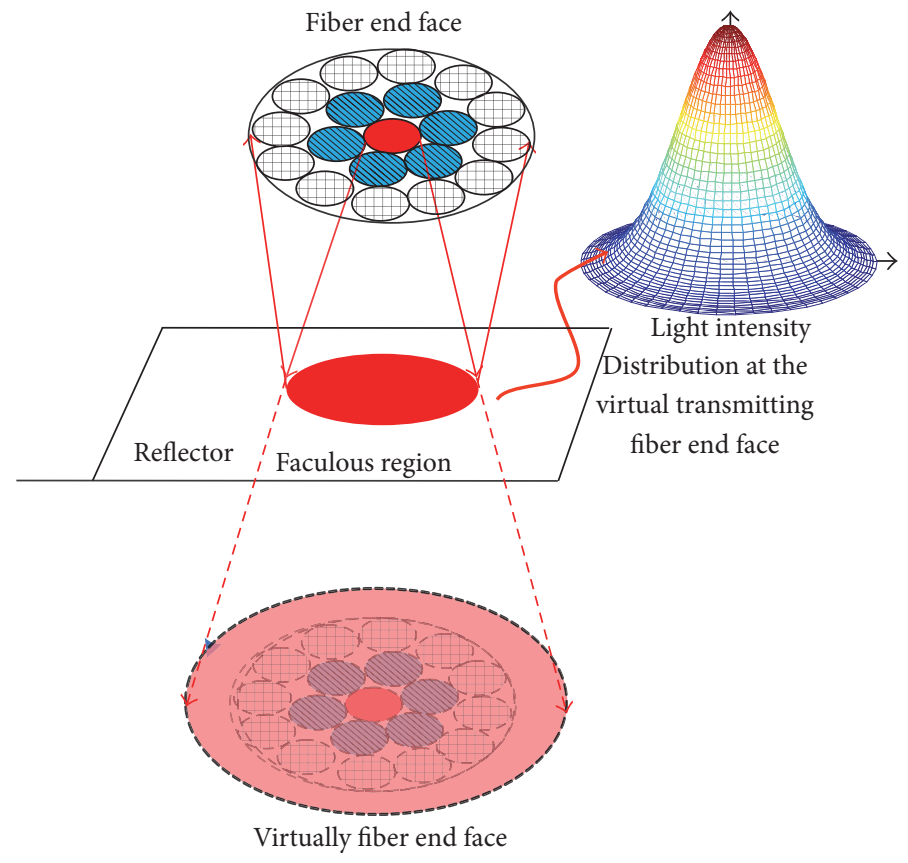

(b) Principle of reflective light intensity-modulated

FIgURE 4: Structure of the reflective intensity-modulated optical fiber bundle.

2.3. Light Intensity to Voltage Converter and Low-Pass Filter Circuit. Light intensity to voltage converter and the low-pass filter circuit is composed of photovoltaic module, signal multiplication, dark error offset circuit, and low-pass filter circuit, as shown in Figure 5. The main function of photovoltaic module is converting the light intensity signal reflected by the blade tip into a voltage signal. And the OPT101 (BurrBrown) was chosen as the light-to-voltage device in this system, as OPT101 has a very large measurement area that collects a significant amount of light and thus allows for highsensitivity measurements. The internal feedback resistor is laser trimmed to $1 \mathrm{M} \Omega$. Using this resistor, the output voltage responsivity, $\mathrm{RV}$, is approximately $0.45 \mathrm{~V} / \mu \mathrm{w}$ at $650 \mathrm{~nm}$ wavelength. The dark errors in the electrical characteristics table include all sources. The OPT101 voltage output is $7.5 \mathrm{mV}$ $\mathrm{dc}$ with no light and increases with increasing illumination. In order to trim the dark output voltage to zero, a low-impedance offset driver (op amp) was used to drive pin 8 (Common) of OTP101 because this node has signal-dependent currents [20].

The signal multiplication circuit was designed to improve the signal-to-noise ratio and ensure the accuracy of sampling data. As Figure 5 shows, the operational amplifier INA118 was chosen in this system.
Low-pass filter module is designed for antialiasing filter. According to the sampling theorem, in order to avoid frequency aliasing, the sampling frequency must be as large again as the highest signal frequency or larger. The max RPM is $6000 \mathrm{r} / \mathrm{min}$; the cutoff frequency is set to $30 \mathrm{kHz}$, which is 10 times the highest frequency of the rotor.

\section{Experimental Setup}

To verify the performance of the optical fiber tip clearance measurement system, a measurement experiment system was built (shown in Figure 6). In Figure 6, we have the following: (1) the rotor test-platform (the speed range of the rotor testplatform was from 0 to $6000 \mathrm{rpm}$ ), (2) the rotor (with blades), (3) the optical fiber bundle, (4) the main signal processing unit, (5) the high-speed data acquisition card (USB-1901, ADLINK), (6) computer, (7) the DC power supply (MCH$305 \mathrm{D}$ ), and (8) the laser light source (type: JW3105B, wavelength: $650 \mathrm{~nm}$, and power: $10 \mathrm{~mW}$ ).

The speed adjustable range of the rotor test rig is 0 $6000 \mathrm{r} / \mathrm{min}$. In dynamic simulation measurement experiment, the system dynamic characteristic was verified by adjusting the rotor rotational speed. The blade tip clearance data was acquired using optical fiber displacement probes 


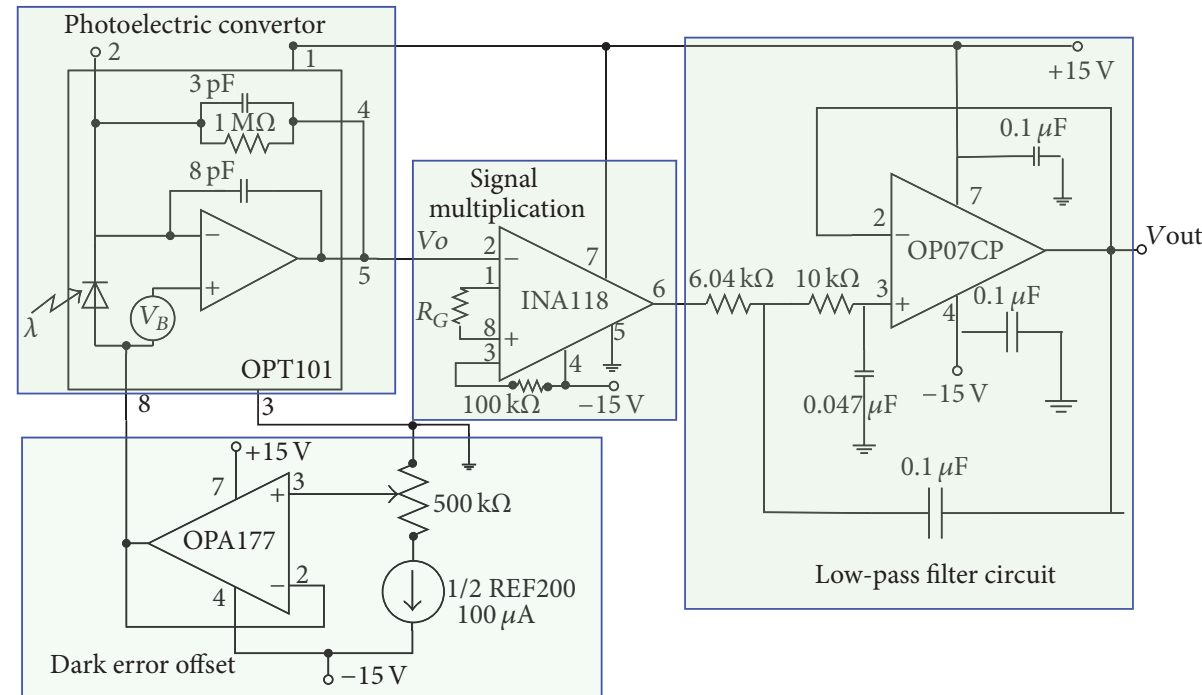

FIgURE 5: Light intensity to voltage converter and Low-pass filter circuit.

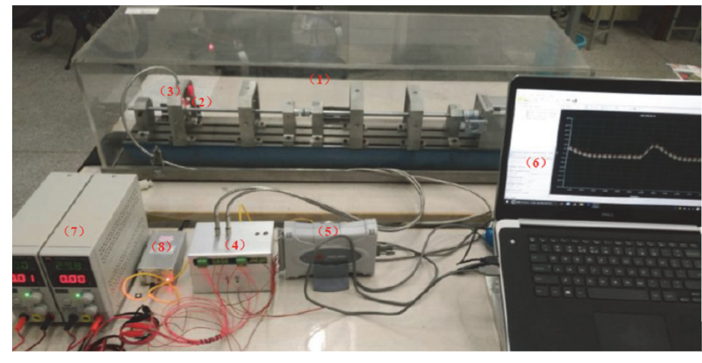

Figure 6: Optical fiber tip clearance measurement system test rig.

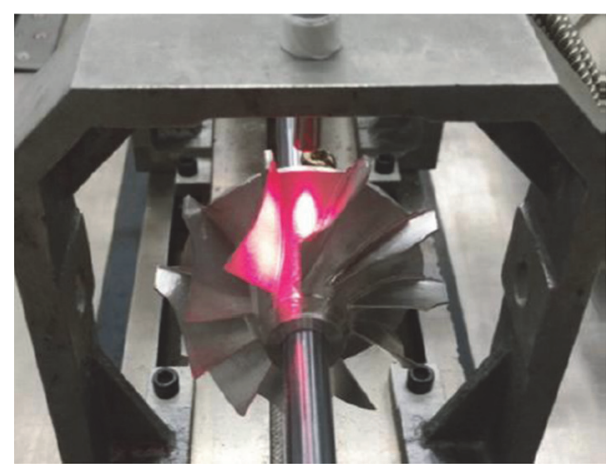

FIGURE 7: Simulated rotor and optical fiber displacement sensor.

as shown in Figure 7. As Figure 7 shows, the rotor had an outside diameter of $90 \mathrm{~mm}$. The thinnest portion of the rotor's web was $2.5 \mathrm{~mm}$. Ten blades were spaced evenly around the circumference of the disk. Each blade had a cross section of $15 \mathrm{~mm}$ by $0.8 \mathrm{~mm}$ and a height of $15 \mathrm{~mm}$.

The displacement calibration platform was used to calibrate the fiber bundle as shown in Figure 8. The platform is composed of a digital micrometer (distance change), a

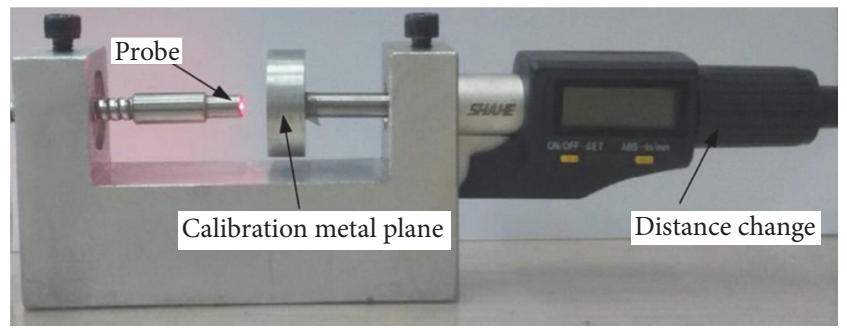

FIGURE 8: Displacement calibration platform.

calibration metal plane, and a metal fixed platform, and the accuracy is $0.001 \mathrm{~mm}$.

\section{Results and Discussion}

\subsection{Static Performance of the System}

4.1.1. Measurement Range and Accuracy and Sensitivity. The calibration components shown in Figure 8 have been used for gaining the measurement range and accuracy of the sensor. During the calibration process, the optical fiber bundle was fixed to the end of the calibration platform by a bolt; the reflector was manipulated moving $25 \mu \mathrm{m}$ in one step in the range of $0 \sim 10 \mathrm{~mm}$. Using this procedure, the calibration curve (shown in Figure 9) of the sensor was obtained. As Figure 9 shows, the sensitivity and linear range are obtained by fitting the experimental $\mathrm{RFa} / \mathrm{RFb}$ to a linear expression with $99 \% R$ square value, the ratio-displacement curve of the receiving fiber bundles RFa and RFb has good linearity in the $3 \sim 8 \mathrm{~mm}$ range, and it can be seen from Figure 9 that its sensitivity is $0.0733 / \mathrm{mm}$. We can see that the sensitivity has very little changes in the measurement range of $3 \sim 8 \mathrm{~mm}$ as well.

4.1.2. Stability Test. A long-term stability experiment was conducted; in the experiment process, the fiber probe was 


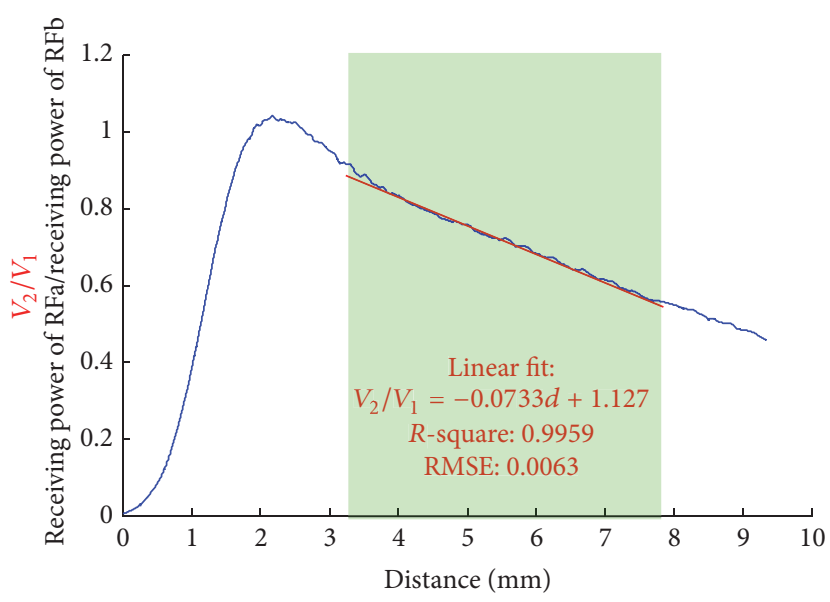

FIGURE 9: Displacement measurement calibration.

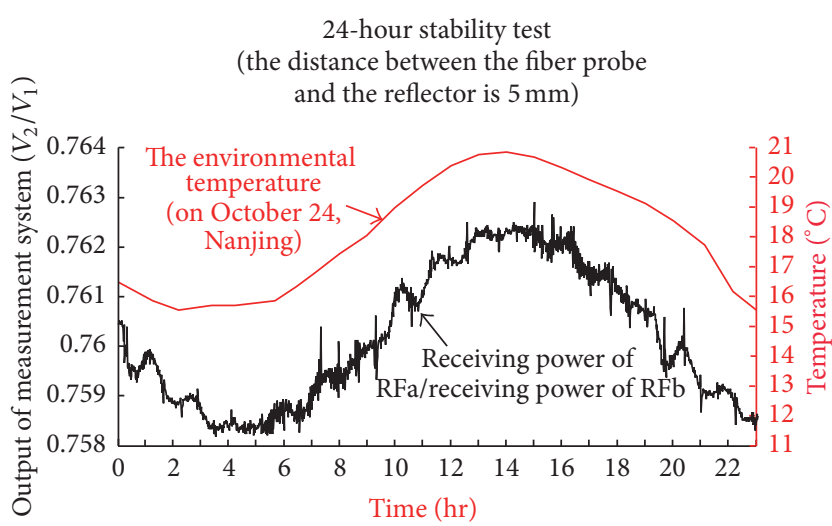

FIgURE 10: Measurement system stability test result.

fixed $5 \mathrm{~mm}$ away from reflector. In our experiment, both the output of the measurement system and the environmental temperature of testing system were acquired as a function of time, and the data were collected for $24 \mathrm{~h}$. The results are shown in Figure 10. We can see that the output of the system drifts with time, and the drift is strongly correlated with the environmental temperature, although this drift is very small. This can be explained by the fact that the probe expands/ contracts in dimension when temperature changes.

4.2. Dynamic Performance of the System. Taking into account the fact that our measurement system's linear range is 3 $8 \mathrm{~mm}$ and the vibration changes range of our rotor test rig between 0 and $2.5 \mathrm{~mm}$, the preinstalled initial distance between the blade tip and the fiber probe is near $5.5 \mathrm{~mm}$, as shown in Figure 7. As shown in Figure 11, the curve shows the filtered signals from the photodetectors and their quotient at a working point of 0 RPM. Based on the calibration results of the optical fiber sensor as shown in Figure 9, we obtain the real initial distance of each blade tip from the optical fiber probe, as shown in Figure 12. In Figure 12, the black number is the maximum distance of blade tip pass through sensor probe; the red number is the minimum distance of blade tip pass through sensor probe. From Figure 12, we can see that the

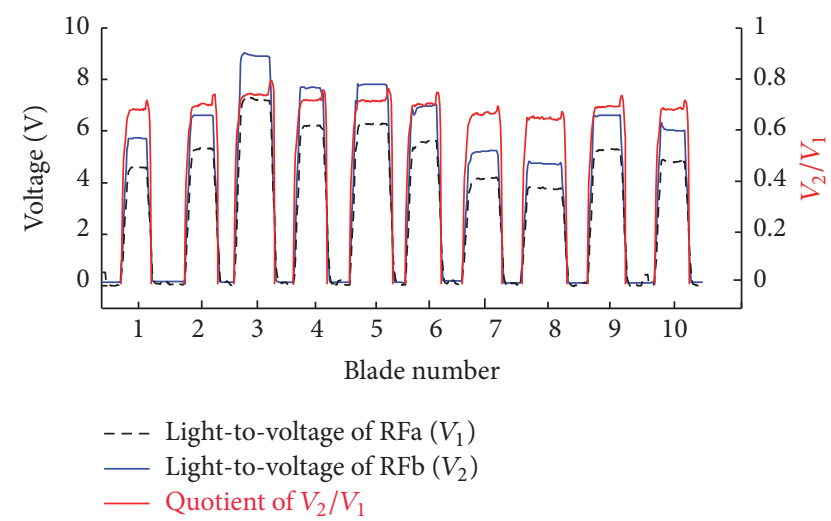

FIGURE 11: Filtered signals from the photodetectors and their quotient at a working point of $0 \mathrm{RPM}$.

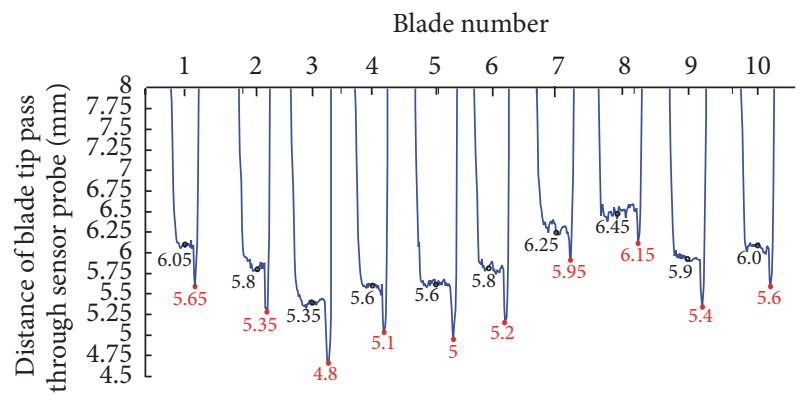

FIGURE 12: The real initial distance of each blade tip from the optical fiber probe at 0 RPM.

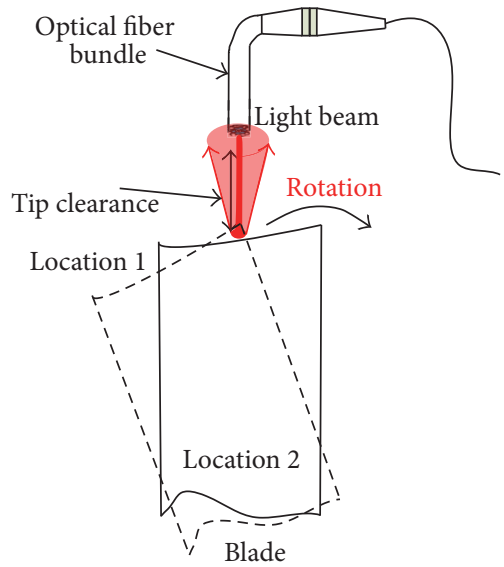

FIgURE 13: The front view when the blade passes through the probe.

initial distance of every blade is different; what is more, the tip clearances of all blades have the same change phenomena when the blade passes through the front of the optical fiber probe. This can be explained by the blade tip surface with a small tilt angle; the geometry relationship of blade tip via the probe was shown in Figure 13.

The typical dynamic outputs (filtered signals data) from the photodetectors as they pass under the optical fiber probe on the simulated rotor spinning at 500, 1000, 2000, 3000, 4000 , and $5000 \mathrm{rpm}$ are shown in Figure 15. For above 

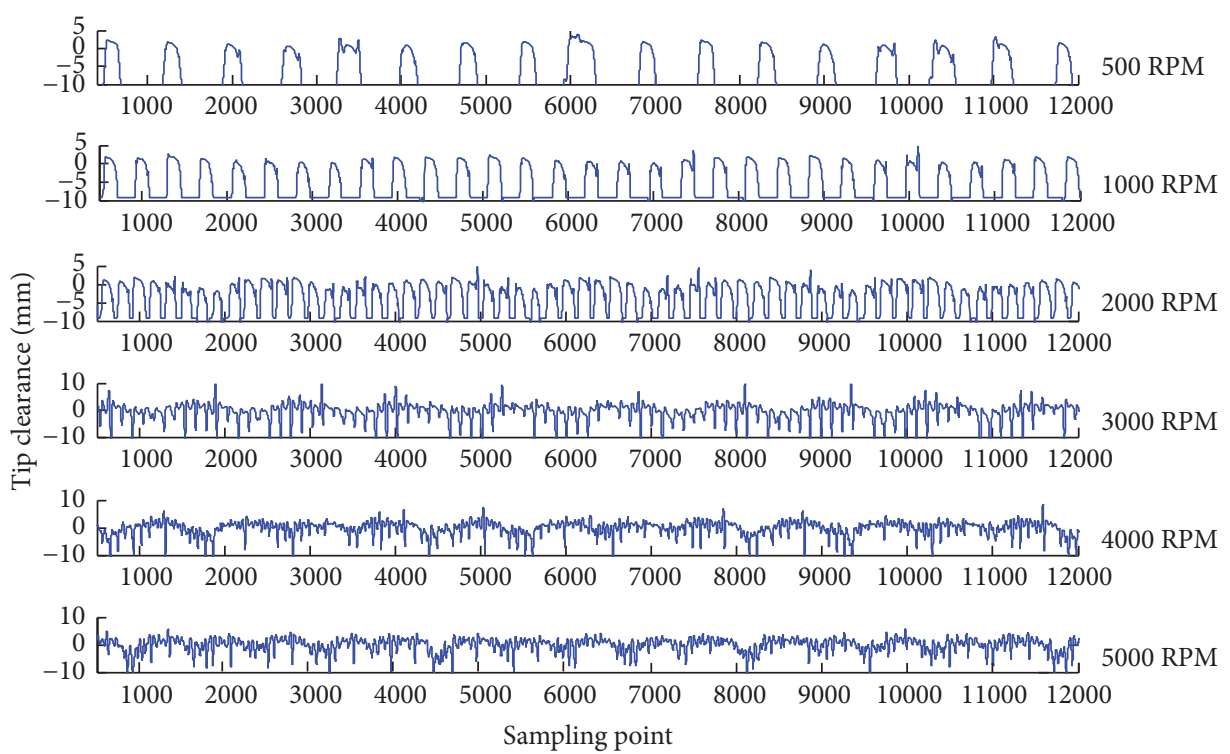

FIGURE 14: The raw filtered signals data from the photodetectors at different working point.

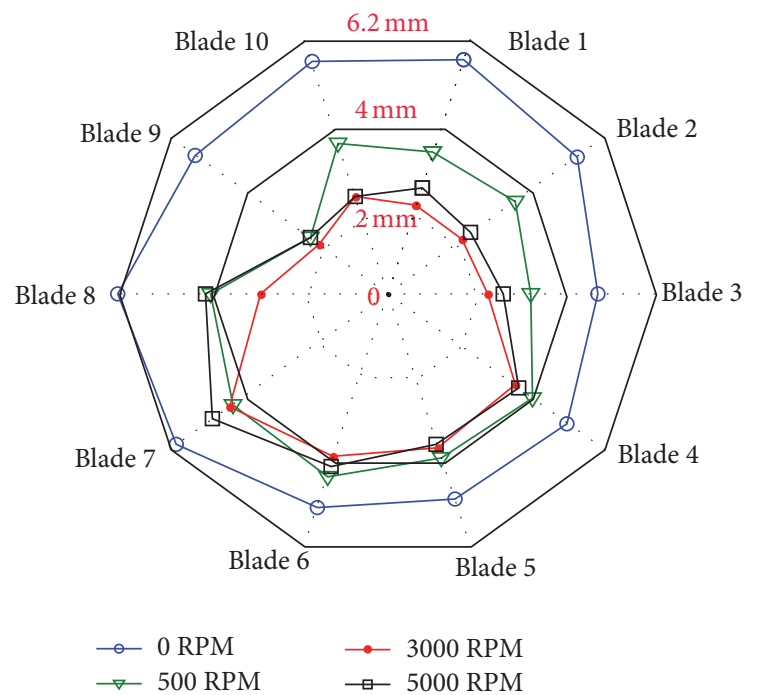

FIGURE 15: Tip clearance measured at different rotor working speed.

rotational speed, the passing frequencies of the rotate blade are $83 \mathrm{~Hz}, 166 \mathrm{~Hz}, 334 \mathrm{~Hz}, 500 \mathrm{~Hz}, 667 \mathrm{~Hz}$, and $833 \mathrm{~Hz}$, respectively. Based on sampling theorem, the sampling frequency should be 2 to 5 times the signal being measured; as Figure 14 shows, our test system could acquire the blade tip profile completely at above speed.

An additional means of viewing the data is shown in the polar plots of Figure 15 and each blade tip clearance changes curve with rotational speed increasing was shown in Figure 16. As noted previously, these plots show that the blade tip clearances decrease as the fan speed is increased. A more noticeable change in the individual clearances can be observed in the polar plot in Figure 15. With the rotation speed increasing, the rotor vibration amplitude is larger; and

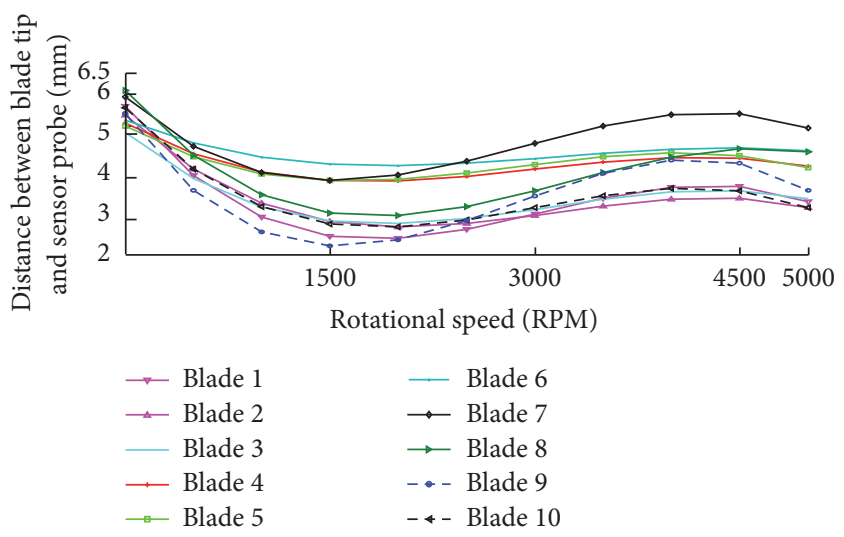

FIGURE 16: The tip clearance of each blade with rotational speed increasing.

the tip clearance has been smaller when working 3000 RPM as the speed close to the critical speed of the rotor test rig. With the speed across the natural frequency of the rotor, the tip clearance slightly increased (shown as Figure 15; the curve of $5000 \mathrm{RPM}$ ).

\section{Conclusion}

In this paper, we propose an optical fiber tip clearance system. The optical fiber tip clearance measurement system consists of the reflective intensity-modulated optical fiber bundle, main signal processing unit, high-speed data acquisition card, and a computer. The structure of the fiber bundle was designed based on the tip clearance change mechanism; and then the principle of the reflective light intensity-modulated function of the fiber bundle we designed was obtained. The light intensity to voltage converter and filter circuit were given. 
In order to verify the performance of the system we designed, the static performance and dynamic performance measurement experiments were done on a simulated rotor test rig. The results show that the accuracy of the system is $25 \mu \mathrm{m}$ or better; the clearance measurement range is about $5 \mathrm{~mm}$, and sensitivity of the sensor is $-0.0733 / \mathrm{mm}$. Furthermore, the typical tip clearance dynamic measurement experiment results show that the system has good dynamic response characteristics as well.

\section{Competing Interests}

The authors declare that they have no competing interests.

\section{Acknowledgments}

National Natural Science Foundation of China (51405222 and 51606092) supported this project.

\section{References}

[1] S. B. Lattime and B. M. Steinetz, "High-pressure-turbine clearance control systems: current practices and future directions," Journal of Propulsion and Power, vol. 20, no. 2, pp. 302-311, 2004.

[2] I. García, J. Beloki, J. Zubia, G. Aldabaldetreku, M. Asunción Illarramendi, and F. Jiménez, "An optical fiber bundle sensor for tip clearance and tip timing measurements in a turbine rig," Sensors (Switzerland), vol. 13, no. 6, pp. 7385-7398, 2013.

[3] K. L. Miller, N. L. Key, and R. D. Fulayter, "Tip clearance effects on the final stage of an HPC," in Proceedings of the 45th AIAA/ASME/SAE/ASEE Joint Propulsion Conference and Exhibit, AIAA, Denver, Colo, USA, August 2009.

[4] L. Neuhaus and W. Neise, "Active control to improve the aerodynamic performance and reduce the tip clearance noise of axial turbomachines," in Proceedings of the 11th AIAA/CEAS Aeroacoustics Conference (26th AIAA Aeroacoustics Conference), Monterey, Calif, USA, May 2005.

[5] A. Vakhtin, S. Chen, and S. M. Massick, "Optical probe for monitoring blade tip clearance," in Proceedings of the 47th AIAA Aerospace Sciences Meeting Including The New Horizons Forum and Aerospace Exposition, Orlando, Fla, USA, January 2009.

[6] G. R. Sarma and J. P. Barranger, "Capacitance-type blade-tip clearance measurement system using a dual amplifier with ramp/DC inputs and integration," IEEE Transactions on Instrumentation and Measurement, vol. 41, no. 5, pp. 674-678, 1992.

[7] A. G. Sheard and B. Killeen, "Blade-by-blade tip clearance measurement system for gas turbine applications," Journal of Engineering for Gas Turbines and Power, vol. 117, no. 2, pp. 326-331, 1995.

[8] A. G. Sheard, "Blade by blade tip clearance measurement," International Journal of Rotating Machinery, vol. 2011, Article ID 516128, 13 pages, 2011.

[9] Z. Long, H. Pengzhou, W. Liang, and W. Zhenhua, "Research on main influencing factors for tip clearance measurement accuracy based on capacitance method," Measurement and Control Technology, vol. 35, no. 5, pp. 137-140, 2016.

[10] J. Binghui and Z. Xiaodong, "An optical fiber Blade tip clearance sensor for active clearance control applications," Procedia Engineering, vol. 15, pp. 984-988, 2011.
[11] M. R. Woike, J. W. Roeder, C. E. Hughes, and T. J. Bencic, “Testing of a microwave blade tip clearance sensor at the NASA Glenn Research Center," in Proceedings of the 47th AIAA Aerospace Sciences Meeting Including the New Horizons Forum and Aerospace Exposition, Orlando, Fla, USA, January 2009.

[12] I. García, J. Zubia, G. Durana, G. Aldabaldetreku, M. A. Illarramendi, and J. Villatoro, "Optical fiber sensors for aircraft structural health monitoring," Sensors, vol. 15, no. 7, pp. 15494-15519, 2015.

[13] D.-C. Ye, F.-J. Duan, H.-T. Guo, Y. Li, and K. Wang, "Turbine blade tip clearance measurement using a skewed dual-beam fiber optic sensor," Optical Engineering, vol. 51, no. 8, Article ID 081514, 2012.

[14] L. Yuan, "Light source and the optical field formed by an optical fiber end," Optical Communication Technology, vol. 18, no. 1, pp. 54-64, 1994.

[15] X. Zhang, S. Xie, H. Niu, P. Zhang, and B. Jia, "Research on dynamic measurement technology of fiber optic sensors and their development," Journal of Vibration, Measurement and Diagnosis, vol. 35, no. 3, pp. 409-416, 2015.

[16] J. A. Kypuros and K. J. Melcher, "A reduced model for prediction of thermal and rotational effects on turbine tip clearance," NASA/TM 2003-212226, NASA, 2003.

[17] W. Olsson and R. Martin, "B747/JT9D Flight Loads and their effect on engine running clearance and performance deterioration," Nacelle Aerodynamic and Inertial Loads (NAIL)/JT9D Jet Engine Diagnostics Programs NASA CR-165573, 1982.

[18] B. Jia, X. Zhang, and X. Ren, "Effect of rotor on changes phenomena of turbine tip clearance," Journal of Vibration, Measurement and Diagnosis, vol. 32, no. 3, pp. 488-492, 2012.

[19] B.-H. Jia, X.-D. Zhang, and K. Peng, "Dynamic changes rule of aero-engine turbine tip clearance in maneuver flight," Journal of Aerospace Power, vol. 26, no. 12, pp. 2757-2764, 2011.

[20] B. Jia, L. He, G. Yan, and Y. Feng, "A differential reflective intensity optical fiber angular displacement sensor," Sensors, vol. 16, no. 9, article no. 1508, 2016. 


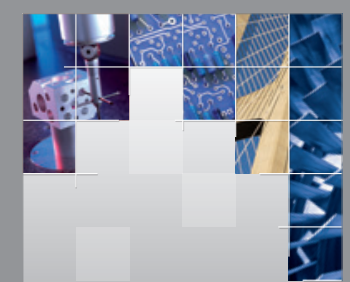

\section{Enfincering}
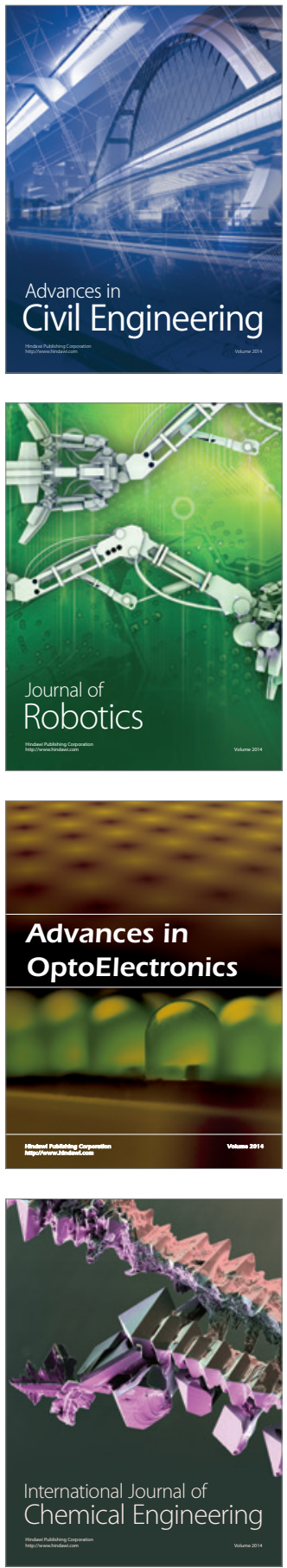

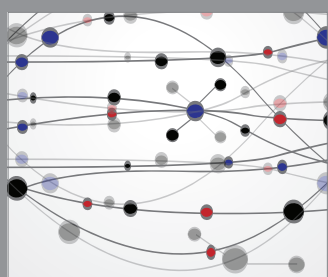

The Scientific World Journal

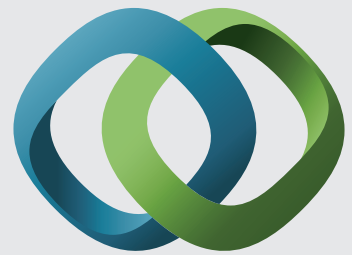

\section{Hindawi}

Submit your manuscripts at

https://www.hindawi.com
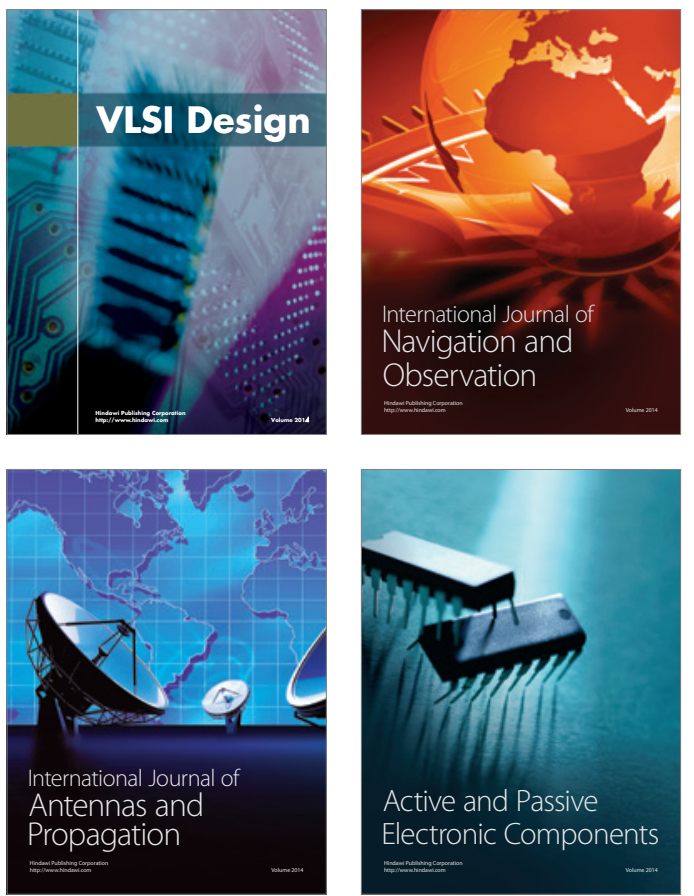
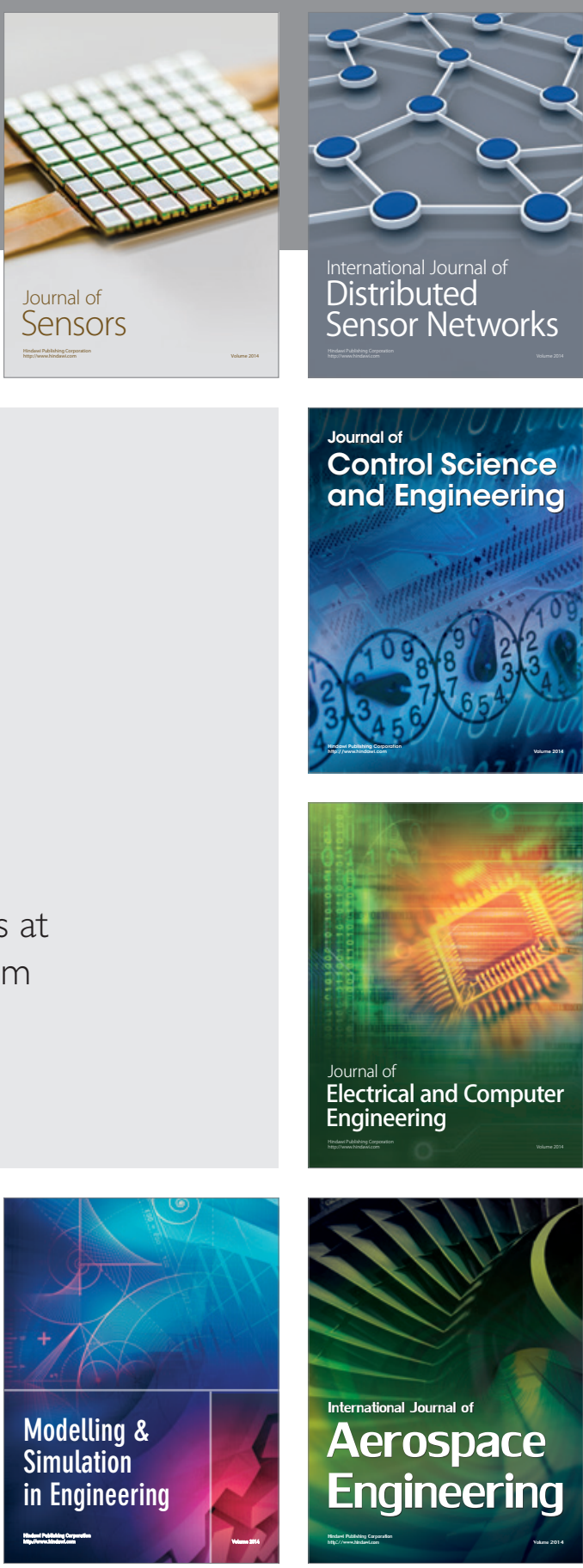

International Journal of

Distributed

Sensor Networks

$-$

Joumal of

Control Science

and Engineering
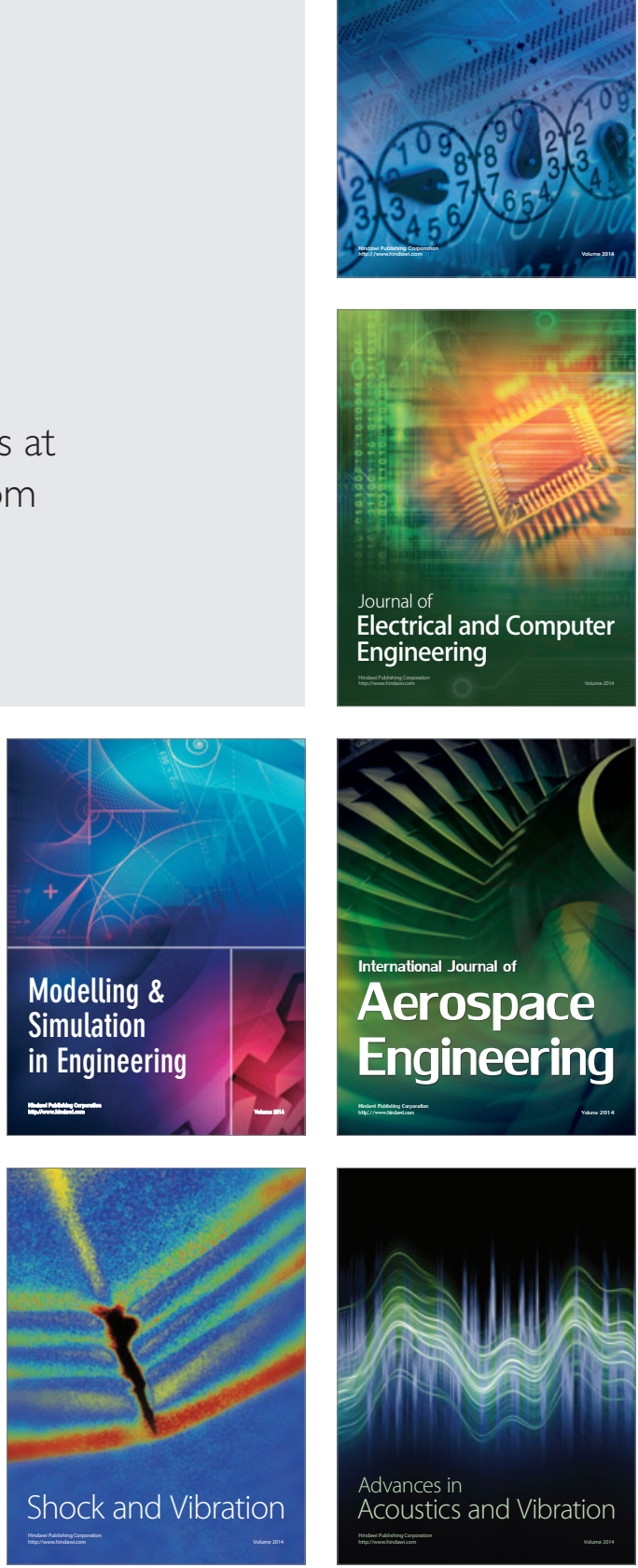